\title{
La consommation urbaine de l'huile de palme rouge en Côte d'Ivoire : quels marchés ?
}

Oléagineux, Corps Gras, Lipides. Volume 8, Numéro 6, 641-5, Novembre - Décembre 2001, Dossier : L'avenir des cultures pérennes

Auteur(s) : Emmanuelle CHEYNS, Cirad, 01 BP 6483 Abidjan 01, Côte d'Ivoire.

Résumé : Au côté d'une extraction industrielle de l'huile de palme dont les débouchés sont l'exportation en Europe et, en Côte d'Ivoire, la seconde transformation en huile industrielle raffinée et produits dérivés (savons, margarines) pour un marché ivoirien et sous-régional, il existe un secteur de transformation artisanal de l'huile rouge (et aussi de l'huile de palmiste et de savons) qui répond à une demande différenciée des consommateurs ruraux mais aussi urbains. En termes d'équipements de transformation, le secteur artisanal est peu développé (beaucoup moins que dans d'autres pays de la sous-région). L'extraction de l'huile rouge y est soit entièrement manuelle, soit semi-mécanisée : utilisation de presses à vis verticales très simples et peu coûteuses. Les trente dernières années de développement industriel n'ont pas permis à la Côte d'Ivoire de se doter d'équipements semiindustriels ou artisanaux, intermédiaires 2 entre l'usine de 20 ou $40 \mathrm{t} / \mathrm{h}$ et la transformation manuelle par les femmes, alors que d'autres pays voisins se sont au contraire dotés de ces équipements [1]. Quoi qu'il en soit, ce secteur artisanal informel, maîtrisé par les femmes (transformation et commercialisation), approvisionne largement les marchés de consommation locaux, ruraux comme urbains, en un produit considéré comme spécifique (que ne fournit pas l'agro-industrie locale) et qui semble toujours apprécié des consommateurs. II nous amène à reconsidérer la filière " palmier à huile " sous l'angle d'une filière dont le produit est aussi « vivrier " (par opposition à produit de " rente ") et qui assure un approvisionnement des marchés locaux, via des circuits informels, en répondant à une demande locale. Une première enquête 3 par entretiens approfondis auprès de 28 ménages abidjanais révèle une différenciation de la demande qui s'établit sur la qualité des produits, et qui justifie l'existence de ce secteur de transformation. Les objectifs de cette enquête étaient de resituer la place de l'huile rouge artisanale dans la consommation des ménages urbains, et les raisons pour lesquelles ce produit n'a pas été entièrement remplacé par l'huile de palme industrielle.

Summary: Since 1963, the development of the palm oil sub-sector in Côte d'Ivoire has been essentially industrial. During this time, however and especially now with privatisation, other production and transformation systems based on diversity continued to exist. The diversity in oil palm extraction systems, in artisanal and informal sectors, corresponds to a segmented demand for red palm oil. A survey on oil consumption, particularly on palm oil, was thus conducted in Abidjan households. Consumers differentiate red oil from selected seed palms, from African palms, and from specific regions in the country, like "Man". The different qualities of red palm oil are reflected by different prices on the consumption market. Red palm oil is still largely consumed and is used in specific meals, in which refined palm oil cannot be used. Quality demands of the consumers for red oil lead to proximity relations with the suppliers and to the development of a specific artisanal production.

Keywords: red palm oil, food consumption, households, informal sub-sector, quality product, Abidjan, Côte d'Ivoire. 


\section{ARTICLE}

\section{Maintien de l'huile rouge dans la consommation alimentaire des urbains}

Contrairement à ce qui est le plus souvent admis ${ }^{4}$, I'huile rouge artisanale est encore largement consommée en milieu urbain, même dans la capitale, Abidjan. L'huile de palme raffinée, décolorée et désodorisée, ne s'est pas complètement substituée à l'huile rouge " traditionnelle " des régions de I'Ouest, du Sud, de l'Est et du Centre. Nous verrons notamment qu'elle s'est substituée à l'huile rouge dans certains plats seulement : la cuisson du riz, les fritures et les grillades.

Parmi les 28 ménages urbains interrogés, un seul ne consomme pas d'huile rouge : il s'agit d'une femme ivoiro-sénégalaise qui a surtout vécu au Sénégal, et qui s'identifie beaucoup plus à la culture sénégalaise qu'ivoirienne, qu'elle rejette en partie. Elle ne consomme pas ce produit, " traditionnel » en Côte d'Ivoire, absent des régimes alimentaires sénégalais. Sur les 27 ménages interrogés qui consomment l'huile rouge, la consommation moyenne est de 1,4 litre/mois, soit 17 litres/an (elle s'étend de 0,3 litre/mois à 5 litres/mois, mais la majorité des ménages en consomment entre 1 et 2 litres/mois).

L'huile de palme raffinée, dans ces ménages, est consommée en quantités quatre fois plus importantes : en moyenne 6 litres/mois, soit 72 litres/an. Aujourd'hui, I'huile de palme raffinée, surtout connue sous une des trois marques ivoiriennes, "Dinor ", fait maintenant partie intégrante de l'alimentation courante des ménages ivoiriens. Pour la plupart des ménages interrogés, c'est "I'huile que consommaient nos parents, ils achetaient Dinor, nous aussi on achète Dinor ". L'huile de palme raffinée est l'huile la plus couramment consommée ; les autres produits similaires tels que l'huile de soja, de maïs, de tournesol, etc., sont beaucoup moins accessibles (huiles importées, chères et non accessibles au micro-détail sur les marchés ou dans les boutiques, contrairement à l'huile de palme raffinée), sont considérés comme des produits " de luxe " et sont consommés occasionnellement et pour certains plats (utilisation dans des sauces vinaigrettes, par exemple).

Il est difficile de dire si la consommation d'huile rouge en quantité a diminué parallèlement à l'introduction de l'huile raffinée, du fait de l'absence de chiffres anciens. Mais il est certain que l'huile raffinée s'est substituée à l'huile rouge dans certaines préparations. Cela dit, il ne s'agit pas de tous les plats. Les ménagères considèrent l'huile rouge et l'huile " Dinor » comme deux produits différents, non substituables pour la plupart des plats qu'elles préparent ; chacune des deux huiles a ses usages spécifiques et s'incorporent dans des plats différents. D'ailleurs, I'huile raffinée " Dinor " (marque la plus fréquemment citée) n'est pas toujours considérée comme une huile de palme : elle est, dans un tiers des cas, assimilée à de l'huile d'arachide ${ }^{5}$ !

\section{Des huiles pour des plats différents : « de l'huile rouge pour les plats africains »}

L'huile de palme rouge et l'huile de palme raffinée ne sont pas consommées dans les mêmes plats. Le tableau 1 présente les usages de chacune des deux huiles en fonction du plat préparé.

L'huile de palme raffinée est essentiellement utilisée pour la cuisson du riz, les fritures et les grillades. Dans la cuisson du riz blanc (riz cuit dans l'eau avec des oignons) ou du " riz-gras " (riz cuit dans la sauce), elle permet au riz de ne pas coller. L'huile raffinée est ensuite utilisée pour la plupart des fritures - pommes de terre frites, igname frite, viandes et poisson, œufs, allocos (banane plantain frite) -, et pour les grillades des viandes et poissons. Elle intègre aussi certaines sauces dans lesquelles « on ne met pas l'huile rouge ", mais toujours pour faire frire la viande en début de préparation (" sauce 
tomate ", " sauce pistache », " sauce aubergine », " sauce claire »).

L'huile rouge entre dans la composition des sauces " africaines" (les "sauces feuilles " et les sauces au gombo - secs et frais, soit " sauce djumblé » et "sauce Kopé ${ }^{6}$ ") et de plats africains à base de banane plantain, de manioc ou d'igname - foufou (banane ou igname cuit et pilé), sauce spéciale attiéké (manioc), attiéké "rouge ", igname bouilli et allocos -, qui sont pour la plupart des plats courants. À la différence de I'huile raffinée, I'huile rouge est incorporée dans les sauces au gombo comme condiment dans la sauce, et non pour frire la viande ou le poisson. Elle doit lui donner du goût et une couleur particulière. Dans les sauces feuilles, en revanche, I'huile rouge peut servir à frire le poisson ou la viande, tout comme l'huile raffinée, sans pourtant être remplacée par cette dernière. Il y a donc bien des usages spécifiques à chacune de ces huiles.

À la question de savoir si on peut remplacer l'huile rouge par un autre produit dans certains plats, il apparaît que l'huile rouge peut effectivement être remplacée par l'huile de palme raffinée dans les sauces gombo ( 7 cas), sauces feuilles ( 6 cas) ou dans l'igname bouilli (3 cas). Elle ne peut, en revanche, absolument pas être remplacée dans la préparation du foufou banane, plat fréquemment consommé pendant la période de forte production des bananes plantain. Les frontières entre les deux huiles sont ainsi, quand même, relativement souples pour certains plats, et pourraient encore évoluer. Les allocos sont par exemple, aujourd'hui, aussi bien frits dans I'huile raffinée que dans I'huile rouge, et cela dans les mêmes ménages.

Enfin, nous avons demandé aux femmes interrogées s'il y a des plats qu'elles ne préparent plus avec I'huile rouge : cela est apparu dans trois ménages pour un plat donné. Mais parallèlement, dans trois autres ménages, les femmes ont appris de nouvelles recettes qui incorporent l'huile rouge, dont un plat ivoirien et deux plats étrangers africains appris auprès d'une Béninoise et d'une Togolaise (à base de gari, de haricot et d'huile rouge).

Ainsi, l'huile rouge est-elle un aliment spécifique toujours largement consommé en ville, qui trouve sa place dans des plats particuliers, pour lesquels elle ne peut être remplacée par un autre corps gras. Nous verrons qu'elle a des propriétés perçues comme spécifiques par les consommateurs qui la différencient de I'huile raffinée : un goût différent, une couleur rouge, et c'est un produit « de terroir $"$.

Enfin, il faut souligner que le différentiel de prix entre ces deux huiles n'est pas en faveur de l'huile rouge. L'huile rouge artisanale la plus consommée (dite " huile africaine ") est vendue environ 900 FCFA/litre au lieu de 600 FCFA/litre pour I'huile raffinée (tableau 2). L'huile rouge n'est donc pas un produit utilisé « à défaut " de moyens financiers. On ne peut avancer que la quantité de 1,4 litre/mois consommée l'est pour accéder à une huile de palme moins coûteuse que celle qui est raffinée.

\section{Huile rouge artisanale : un produit de terroir, un produit « du village »}

Pour les consommateurs, les deux critères principaux de différenciation des qualités d'huile rouge sont : a) la nature des graines utilisées, à savoir des graines sélectionnées, dites « sodepalm » (du nom de l'ancienne société d'exploitation des ensembles agro-industriels) ou des graines de palmier naturel ou sauvage (dites " graines africaines", "graines de l'homme noir "); et b) le procédé de transformation, le plus souvent identifié par un territoire (région, village, etc.).

En simplifiant, trois types d'huiles sont le plus souvent distingués par les consommateurs d'Abidjan : I'huile " sodepalm », l'huile " africaine », et l'huile " de Man » (ou de l'Ouest). Concernant la région de 
Man, il faut signaler qu'elle est située au Centre-Ouest du pays. Dans cette région, les sociétés d'exploitation du palmier sélectionné ne se sont pas implantées et c'est en même temps une région riche en palmeraies naturelles. II n'y a donc en principe pas de graines sélectionnées ou " sodepalm » dans cette région ${ }^{7}$. Par ailleurs, le procédé de transformation y est entièrement manuel et surtout la fermentation des graines très réduite par rapport à d'autres régions.

Pour distinguer les qualités d'huile rouge, les ménagères font le plus souvent référence à ces trois types d'huile (tableau 3), I'huile " sodepalm » étant considérée à l'unanimité comme étant la moins bonne, et très peu achetée.

Cette opposition entre I'huile " sodepalm » (moins bonne au goût des consommateurs) et l'huile africaine de telle ou telle région, la plus appréciée, et définie comme " la vraie huile ", se traduit par une différence de prix allant du simple au double (490 FCFA pour l'huile " sodepalm », 915 FCFA environ pour l'huile " africaine » ou " de Man » - tableau 2). Les critères les plus souvent utilisés pour définir l'huile rouge de qualité sont : une huile bien rouge (et non orange), qui ne " tire " ou ne " gratte " pas sur la langue, liquide/limpide/claire (qui " ne dort pas ») et enfin qui se mélange bien dans la sauce (I'huile ne remonte pas à la surface). Or, l'huile « sodepalm » aurait tous les défauts : pas assez rouge (tire sur l'orange), elle " dort " (elle se fige), " tire " sur la langue et ne se mélange pas bien à la sauce. Ces critères sont d'ailleurs ceux éprouvés par les ménagères au moment de l'achat et selon la personne à qui elles achètent, pour définir la qualité de l'huile : regarder sa texture et sa couleur, et surtout la goûter avant achat.

Les différences de qualité du produit fini sont attribuables, pour les ménagères, soit au type de graines utilisées, soit au procédé de fabrication (tableau 4). Les ménagères parlent ainsi de l'huile de graines " sodepalm » et de l'huile africaine, mais aussi de I'huile de Bouaké, I'huile de Gagnoa, I'huile de Bouaflé, I'huile des Adjukru, I'huile des Attiés, etc. Le type de graines a été évoqué par 20 ménagères (sur 27) pour expliquer la qualité de ces huiles. L'huile de certaines régions situées hors des zones d'implantation du palmier sélectionné (Man, mais aussi Gagnoa ou Bouaké, par exemple) est appréciée parce que, dans ces régions, les graines sélectionnées n'existent pas, il s'agit forcément, pour les consommatrices, d'une huile de graines « africaines ». Quant aux modes de préparation, c'est le temps de fermentation des graines qui est le plus souvent invoqué pour expliquer les différences de qualité.

\section{Des modes d'approvisionnement sur la confiance pour une qualité territoriale}

Les modes d'approvisionnement des ménagères sont révélateurs de ces spécificités territoriales. Quasiment toutes ont une relation de proximité avec leur vendeuse ou fournisseur : fidélisation au marché à une femme qui vend l'huile de telle région ou qui est de telle ethnie, achat directement au village ou par l'intermédiaire de parents, achat dans une région spécifique par l'intermédiaire de relations, etc. (tableau 5). Chez un même ménage, les lieux d'achat peuvent être divers, le cas le plus fréquent étant l'approvisionnement en gros de l'huile du village ou par relation, complété par des achats plus ponctuels au marché. Seules deux ménagères ont, en complément, recours à l'achat de I'huile au marché sans être fidélisées ni attachées à un certain type d'huile et trois ménagères disent de temps en temps devoir acheter de I'huile « sodepalm ». En dehors de ces cas, au marché, la plupart des ménagères sont fidélisées à une vendeuse.

Elles achètent alors chez une femme "yacouba ", " adjukru ", " attié ", " gouro ", etc., ou l'huile de telle région. Il faut souligner qu'il ne s'agit alors pas nécessairement d'une femme de même ethnie que 
l'acheteur. Dans ce cas, ce n'est pas l'huile de sa propre région d'origine qui est recherchée, mais celle d'une région donnée. II en est de même pour les neuf personnes qui, au marché, achètent l'huile de Man, et qui ne sont pas des femmes originaires de cette région. Enfin, un tiers des ménagères s'approvisionnent au village. Dans ce cas, il peut s'agir de dons mais pas nécessairement. Pour les ménagères qui s'approvisionnent au village, cette huile ne peut être suspectée, c'est nécessairement une huile « de très bonne qualité ».

Ces particularités de l'approvisionnement en huile soulignent l'ancrage territorial de ce produit. Mais ces procédures particulières sont aussi un moyen de se garantir la qualité. La grande diversité des huiles vendues sur les marchés, quant à leur apparence (différents degrés de couleurs, différents modes de décantation, parfois difficultés à en évaluer le goût, suspicion de fraude par les vendeuses qui mélangeraient « l'huile sodepalm » à « l'huile africaine » pour augmenter leur bénéfice, etc.), rend parfois les achats incertains. L'approvisionnement chez des parents du village, la proximité établie avec une vendeuse, ou l'identification d'un réseau d'approvisionnement par relation, sont des procédures de qualification du produit [2,3]. Ces procédures sont fondées sur la confiance entre des personnes qui se connaissent [4] ou qui établissent un lien de fidélisation.

L'huile de Man bénéficie d'une réputation particulière, comme s'il s'agissait d'un label de qualité, qui dépasse la proximité territoriale entre vendeur et acheteur ; ce sont des ménagères de toutes origines qui s'approvisionnent en huile de Man.

D'autres procédures de qualification ont été mises en évidence. L'achat auprès d'une " vieille " est parfois justifié par une garantie sur la qualité du produit. La garantie réside dans le fait que les " vieilles " ont un savoir-faire expérimenté, mais aussi qu'elles " n'ont plus trop cette idée de gagner de l'argent, elles ne vont pas faire des mélanges " (entretien avec une consommatrice) à l'insu de l'acheteur, l'anecdote étant cette ménagère qui précise " qu'on ne sait jamais, on dit tellement de choses, parfois même il paraît que des vendeuses mettent des mille-pattes ${ }^{8}$ dans I'huile ".

Ces résultats indiquent ainsi l'existence d'une demande locale en huile rouge artisanale dont la qualité est, pour les acheteurs, intimement liée à la nature des graines et/ou à des procédés de fabrication locaux. Le secteur de transformation artisanal, appuyé sur des savoir-faire familiaux et des réseaux de distribution personnalisés, répond à cette demande. Ce secteur mériterait une attention particulière en termes de recherche-développement.

Notes :

${ }^{1}$ Cet article a fait l'objet d'une communication à la conférence internationale " Avenir des cultures pérennes » de Yamoussoukro, Côte d'Ivoire, 5-9 novembre 2001, Bnedt/Cirad/ESI-VU.

${ }^{2}$ Quelques mini-huileries font leur apparition, mais ce phénomène est très récent et leurs marchés ne sont pas encore bien connus.

${ }^{3}$ Ces travaux s'inscrivent dans le cadre du projet de recherche sur « l'organisation et le développement de la filière palmier à huile en Côte d'Ivoire » conduit par le Cirad et l'Université de Bouaké. L'enquête a été réalisée en avril 2001.

${ }^{4}$ Le discours des opérateurs de la filière en Côte d'Ivoire est fréquemment celui de la disparition de la consommation d'huile rouge dans les ménages, qui serait en partie provoquée par l'urbanisation. 
${ }^{5}$ Les autres façons de définir l'origine de l'huile " Dinor » (ou d'autres marques d'huile de palme raffinée) ont été : a) " fabriquée à partir de l'huile de palme rouge ", dans 8 cas dont : " par chauffage ", par adjonction de "produits chimiques ", ou par " raffinage "; b) à partir de l'huile de palmiste (2 cas) ; c) issue du broyage de la noix de palme entière (1 cas) ; et d) ne sait pas comment est fabriquée cette huile ou de quel produit on la tire (6 cas).

${ }^{6}$ En langue agni - baoulé. Cela est de moins en moins vrai. Depuis quelques années, certains planteurs auraient introduit des semences sélectionnées.

${ }^{8}$ Pour remuer l'huile et l'empêcher de se figer.

\section{CONCLUSION}

Le développement agro-industriel du palmier à huile a permis au pays de se doter d'une production locale de corps gras, et notamment d'approvisionner le marché local en huile raffinée à des prix moins chers que les huiles importées (arachide, tournesol, maïs, etc.). À côté de ce secteur dominant, il existe cependant toujours une demande en huile rouge artisanale, qui s'accorde finalement assez bien avec des formes de production moins intensives.

S'il semble bien que des modèles de production-transformation-valorisation alternatifs au modèle agro-industriel existent (voir l'article de Kouamé et Akindès, dans ce même numéro), se maintiennent, voire se développent, la question qui se pose est de savoir comment ils s'articulent entre eux et avec le reste de la filière et ce qu'ils supposent en termes de règles d'organisation.

Une étude ciblée sur la transformation artisanale devrait permettre de mieux établir le lien entre la demande locale en huile rouge et certaines formes de production du palmier. Il est possible que ce que les consommatrices présentent comme "l'huile africaine " soit aujourd'hui préparée avec des graines sélectionnées, mais selon des procédés particuliers qui correspondent aux exigences de qualité des consommateurs en termes de produit fini. Les systèmes de transformation artisanaux ont pu évoluer en même temps que l'introduction des nouvelles semences.

D'une manière générale, ce marché local de l'huile rouge artisanale est très mal connu. Certaines pistes concernant ses débouchés en consommation alimentaire sont données ici, mais devraient être validées sur un plus large échantillon, au moins pour connaître la taille de ce marché. De même, le marché de l'huile rouge destinée à la fabrication des savons artisanaux semble important ; de nombreuses savonneries artisanales s'installent en ville. Dans une dynamique d'industrialisation de la filière, les secteurs artisanaux ont été très peu développés, si ce n'est depuis cinq ans avec l'introduction de la presse. Pourtant, l'existence d'un marché local du savon et de l'huile rouge artisanale pour la consommation pourrait justifier des recherches et des projets d'amélioration ou d'échanges d'expériences en matière de procédés d'extraction et d'organisation de ces acteurs, pour une culture qui manifestement n'est pas qu'une culture de rente, mais aussi une culture vivrière avec des débouchés locaux. 


\section{REFERENCES}

1. ROUZIÈRE A (1995). Étude mini- et micro-huilerie de palme en Afrique de l'Ouest et du Centre. Rapport de mission Cirad-CP/Caisse française de développement, 2 vol.

2. BRICAS N, CHEYNS E (1995). Incertitudes et procédures de qualification des consommateurs urbains au Burkina. Le cas d'un condiment alimentaire traditionnel : le soumbala. Séminaire Qualification des produits et des territoires, Toulouse, 2 et 3 octobre 1995, INRA, 16 p.

3. CHEYNS E (2001). La qualification des produits artisanaux, du marché de proximité à l'échange à distance. Le soumbala au Burkina Faso. In : MOITY-MAÏZI P, SAINTE MARIE C (de), CIESLIN P, MUCHNIK J, SAUTIER D, eds. Systèmes agroalimentaires localisés, terroirs, savoir-faire, innovations. Étud Rech Syst Agraires Dév 32 : 65-78.

4. SERVET JM (1994). Paroles données : le lien de confiance. Revue du MAUSS : À qui se fier ? Confiance, interaction et théorie des jeux, $4: 37-56$.

5. ORLÉAN A (1994). Sur le rôle respectif de la confiance et de l'intérêt dans la constitution de l'ordre marchand. Revue du MAUSS : À qui se fier ? Confiance, interaction et théorie des jeux, 4 : 17-36.

Illustrations

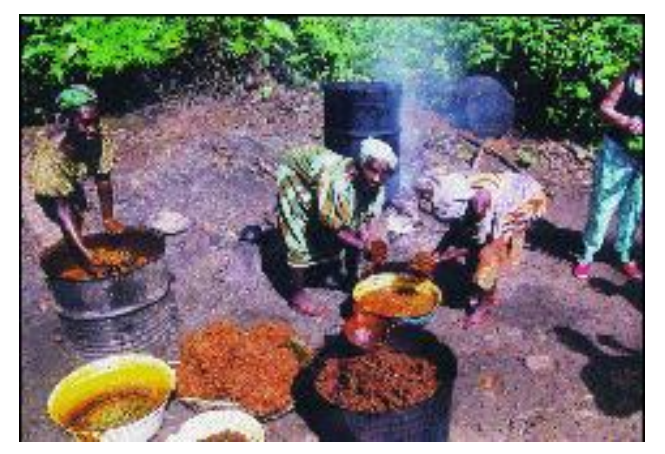

Photo. Transformation artisanale manuelle dans la région de Man. 
Tableau 1. Nombre de plats* incorporant de l'huile de palme rouge et raffinée.

\begin{tabular}{|c|c|c|}
\hline Plats & $\begin{array}{c}\text { Incorporant } \\
\text { I'huile } \\
\text { de palme } \\
\text { rouge }\end{array}$ & $\begin{array}{l}\text { Incorporant } \\
\text { I'huile } \\
\text { de palme } \\
\text { raffinée }\end{array}$ \\
\hline $\begin{array}{l}\text { Sauce Djumblè } \\
\text { (gom bo sec) }\end{array}$ & 21 & \\
\hline $\begin{array}{l}\text { Sauce Kopé } \\
\text { (gom bo fraiss) }\end{array}$ & 10 & 1 \\
\hline Foufou & 21 & \\
\hline Sauce feuilles & 11 & 2 \\
\hline lgname bouilli & 5 & 1 \\
\hline Attiéké rouge & 9 & \\
\hline Sauce d'attiéké & 4 & \\
\hline $\begin{array}{l}\text { Albo (banane } \\
\text { plantain frite) }\end{array}$ & 5 & 4 \\
\hline Fritures & & 11 \\
\hline Grillades & & 14 \\
\hline Riz-gras & 1 & 14 \\
\hline Riz blanc & 2 & 17 \\
\hline Sauce tomate & & 14 \\
\hline Sauce pistache & & 3 \\
\hline Sauce aubergines & & 5 \\
\hline Sauce arachide & & 3 \\
\hline Sauœe claire & 3 & 5 \\
\hline Spag hettis & & 1 \\
\hline Soumbalalafri & 2 & \\
\hline
\end{tabular}

- Cités pa les 28 mérageres, à la question a darr quek plats utilisez-vas l'huile rougg//'huile raffinè? ? s.

Tableau 2. Prix des différentes huiles à Abidjan (mas-avil 2001).

\begin{tabular}{|c|c|c|c|}
\hline Huile de palme & Prix & Autres huiles industrielles & Prix \\
\hline Huile rouge artisanale $\approx$ de Man » & 920 (moyenne) & Huile de soja & 1360 \\
\hline Huile rouge artisanale $\alpha$ africaine » & 910 (moyenne) & Huile de tournesol & 1490 \\
\hline $\begin{array}{l}\text { Huile rouge } \text { * sodepalm » } \\
\text { pour la consommation }\end{array}$ & 490 (moyenne) & Huile d'arachide & 1990 à 2300 \\
\hline Huile industrielle raffinée $\propto$ Dinor $»$ & 600 à 650 & Huile de maiss & 1 600à 1800 \\
\hline Huile industrielle raffinée $\propto \mathrm{Pa} I m$ d'Or » & 600 à 650 & Huile d'olive & 4000 à 7000 \\
\hline
\end{tabular}

Kerrarque : par les prix de l'huile rauge a tisanale et * sodepalm s, vendes sur les machés : prix moyens sur 15 machés pris dars les 10 ommunes d'A bidjan. 
Tableau 3. Critères de differenciation des huiles rouges par kes consommatices.

\begin{tabular}{|c|c|}
\hline $\begin{array}{l}\text { Différentes qualité } \\
\text { d'huile rouge }\end{array}$ & $\begin{array}{l}\text { Nombre } \\
\text { de réponses }\end{array}$ \\
\hline Huile $\propto$ sodepalm » & 21 \\
\hline Huile \& de Man » & 16 \\
\hline $\begin{array}{l}\text { Huile de } \alpha \text { graines africaines } \$ \\
\text { de } \propto \text { nos graines villageo ises } \$\end{array}$ & 13 \\
\hline $\begin{array}{l}\text { Huile de } \text { ж telle région » } \\
\text { (hors zone palmier sodepalm) }\end{array}$ & 6 \\
\hline $\begin{array}{l}\text { Huile de } * \text { telle rigion } \$ \\
\text { (zone palmier sodepalm) }\end{array}$ & 4 \\
\hline $\begin{array}{l}\text { Huile } \propto \text { qui sent mauvais } * / \\
\text { \&raie huilequi nedort } p a s \$ \text {, etc. }\end{array}$ & 2 \\
\hline Huile $\approx$ dioula $»$ & 1 \\
\hline Huile $\approx$ togolaise $»$ & 1 \\
\hline $\begin{array}{l}\text { Huile } \propto \text { artisanale } \approx \\
\text { huile semi-industrielle } \\
\text { la } \propto \text { villageoise } \$\end{array}$ & 1 \\
\hline
\end{tabular}

Tableau 4. Griteres quicondtionnent la qualité de l'huie rouge par la consommation (entretiens ménagers).

\begin{tabular}{|l|c|}
\hline $\begin{array}{l}\text { Bonne qualité } \\
\text { de I'huile liée }\end{array}$ & $\begin{array}{c}\text { Nonbre } \\
\text { de réponses }\end{array}$ \\
\hline $\begin{array}{l}\text { Au fait que dans œtte région } \\
\text { il n'existe pas de graines } \\
\alpha \text { sodepalm », donc œ sont } \\
\text { foroement des graines africaines } \\
\begin{array}{l}\text { Au type de gra ine: } \\
\text { graines africaines }\end{array}\end{array}$ & 8 \\
$\begin{array}{l}\text { Au temps de fermentation } \\
\text { de la graine } \\
\text { A la manière de préparer } \\
+ \text { au type de graine (africaines) } \\
\text { A la non-utilisation d'engrais } \\
\text { sur les palmiers }\end{array}$ & 6 \\
\hline
\end{tabular}


Tableau 5. Lieux d'approvisionnement en huile rouge.

\begin{tabular}{|c|c|}
\hline $\begin{array}{l}\text { Lieux d'achat } \\
\text { ou d'approvisionnement }\end{array}$ & $\begin{array}{c}\text { Nombre } \\
\text { de réponses }\end{array}$ \\
\hline $\begin{array}{l}\text { Achat au marché d'une huile } \\
\text { de I'Ouest (ou de Man) }\end{array}$ & 9 \\
\hline 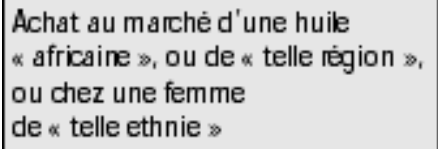 & 10 \\
\hline $\begin{array}{l}\text { Approvisionnement en une huile } \\
\text { d'une autre région par des relatiors }\end{array}$ & 5 \\
\hline $\begin{array}{l}\text { Approvisionnement en une huile } \\
\text { \& de Man } » \text { par des relations }\end{array}$ & 4 \\
\hline $\begin{array}{l}\text { Approvisionnement en huile } \\
\propto \text { de mon village } \text {. }\end{array}$ & 10 \\
\hline Achat chez une $\approx$ voisine & 3 \\
\hline $\begin{array}{l}\text { Achat au marchè d'une huile } \\
\text { \& sodepalm } \text { \& }\end{array}$ & 3 \\
\hline $\begin{array}{l}\text { Achat au marchè, } \\
\text { peu im porte la vendeuse }\end{array}$ & 2 \\
\hline $\begin{array}{l}\text { Achat } \alpha \text { en brousse } »_{1} \\
\text { brs d'un voyage }\end{array}$ & 1 \\
\hline
\end{tabular}

